\title{
Association of Migraine with Its Comorbidities and Food Specific Immunoglobulin G Antibodies and Inflammatory Cytokines: Cross-Sectional Clinical Research
}

\author{
Zhiming Zhao' \\ Huiwen Jin' \\ Yang Yin' \\ Yanwei Hou' \\ Jingyan Wang' \\ Chunling Tang ${ }^{2}$ \\ Jun $\mathrm{Fu}^{\prime}$
}

'Health Center of Screening and Prevention of Diseases, The First Affiliated Hospital of Harbin Medical University, Harbin, I5000I, Heilongjiang, People's Republic of China; ${ }^{2}$ Department of Psychiatry, The First Affiliated Hospital of Harbin Medical University, Harbin, I5000 I, Heilongjiang, People's Republic of China
Correspondence: Jun Fu

Health Center of Screening and

Prevention of Diseases, The First

Affiliated Hospital of Harbin Medical

University, 23 Youzheng Street, Nangang

District, Harbin, 15000I, Heilongjiang,

People's Republic of China

Tel +8645I85553III

Email fujunyk@hotmail.com
Purpose: The relationship between food allergy caused by food specific IgG antibodies and migraine has received increased attention in recent years. Here, we aimed to evaluate the effects of food specific IgG antibodies on headache, gastrointestinal symptoms, anxiety, depression, sleep disorders, dermatosis, and serum inflammatory cytokines in migraine patients, and to quantitatively assess the effect of IgG levels on the severity of headache and its comorbidities.

Methods: Of 89 migraine patients, those who had one or more food specific IgG antibodies $\geq 50 \mathrm{U} / \mathrm{mL}$ were classified into the $\mathrm{IgG}$ positive group, which was then further divided into subgroups based on differing numbers of food allergens. All other subjects were classified into the IgG negative group. We compared the frequency and severity of migraine, anxiety, depression, sleep disorders, dermatosis, and inflammatory cytokines between groups. A regression model was performed to further assess the effect of overall positive IgG concentration and the mediation effect of inflammatory cytokines.

Results: Participants in the positive IgG group $(n=67)$ were more likely to have longer time elapsed since diagnosis, more frequent and severe migraine, a higher risk of developing anxiety and gastrointestinal symptoms, along with higher IL-6 and TNF- $\alpha$. Subgroups with more food allergens generally had worse conditions as well. After adjusting for the inflammatory cytokines, the effect of IgG was reduced.

Conclusion: Migraine patients with positive food specific IgG antibodies had worse migraine, anxiety, and gastrointestinal symptoms. Inflammatory cytokines partially mediate the causal pathway between food specific IgG antibodies, migraine, and migraine comorbidities.

Keywords: migraine, food, immunoglobulin $\mathrm{G}$ antibody, gastrointestinal symptoms, anxiety, interleukin-6, tumor necrosis factor- $\alpha$

\section{Introduction}

Migraine is a recurrent headache disease often characterized by a pulsing sensation, and is associated with a variety of comorbidities including gastrointestinal disorders, neuropsychiatric disorders (ie, anxiety, depression, suicide ideation) etc. ${ }^{1}$ These symptoms may have a dramatic negative impact on one's quality of life and thus might be classified as a type of disability, especially when symptoms interfere with the person's daily activities. Many epidemiological studies have revealed a high prevalence of migraine and its accompanied disorders, as well as 
a burden on individuals and society. ${ }^{2-4}$ Based on a systematic review involving 19 population-based studies from 17 countries, the aggregate weighted estimates prevalence of definite and suspected migraines over a 12month period was $18.5 \%{ }^{5}$ Migraine attacks can be associated with a variety of factors, including dietary consumption. ${ }^{6,7}$ Allergies mediated by food specific IgG antibodies can cause chronic aseptic inflammation in many systems of the body. ${ }^{8}$ High circulating serum concentrations of some IgG antibodies have been measured in certain atopic individuals. ${ }^{9}$ The relationship between food allergy caused by specific IgG positive food and migraine has received increased attention. ${ }^{10}$ Some scholars have proposed that IgG may be a biomarker used to identify foods related to enhanced inflammatory response in vivo, which has been suggested as a crucial role in migraine mechanism and pathology. ${ }^{11-13}$

Proinflammatory cytokines, interleukin-6 (IL-6) and tumor necrosis factor- $\alpha$ (TNF- $\alpha$ ), are considered to be the inducers of visceral pain; ${ }^{14,15}$ migraine patients have been shown to have higher serum levels of IL- 6 and TNF- $\alpha .^{16,17}$ Interleukin-10 (IL-10) is a potent anti-inflammatory cytokine which has been demonstrated to inhibit the production of inflammatory cytokines and attenuate the inflammatory signaling pathway. ${ }^{18}$ Though the connection between migraine and inflammatory cytokines has been reported, ${ }^{19}$ there are no published studies that quantitatively evaluate the correlation between inflammatory cytokines and food specific IgG antibodies in migraine patients.

The aim of this study was to evaluate the effects of food specific IgG antibodies on headaches, gastrointestinal symptoms, anxiety, depression, sleep disorders, dermatosis, and serum inflammatory cytokines (IL-6, IL-10 and TNF- $\alpha$ ), and to explore the quantitative correlation between the concentration and types of serum positive food specific IgG antibodies and symptom scores and the levels of inflammatory cytokines in migraine patients. The findings from this study may improve the integration of evidence regarding the mechanisms of migraine and provide new insights for migraine prevention strategies and treatment plans.

\section{Methods}

\section{Participants}

This cross-sectional study involved participants with a neurologist-confirmed migraine diagnosis defined by The International Classification of Headache Disorders, 3rd edition (ICHD-3). ${ }^{1}$ All participants were recruited during their visit to the Health Center of Screening and Prevention of Diseases of the First affiliated Hospital of Harbin Medical University, between October 2020 and February 2021. Participants were screened for study eligibility, inclusion criteria were: 1) female or male ages 18 to $62 ; 2$ ) minimum six months' time elapsed since migraine diagnosis; and 3) two or more migraine attacks in the past 30 days. Participants whose headaches were caused by another diagnosed disease were excluded (ie, hypertension, stroke, infectious disease, malignant/benign tumors, mental health disorders), participants were also excluded if they had a peptic ulcer, and currently used immunosuppressive drugs.

The ethics and protocol of this research study were assessed and approved by the Ethics committee of the First Affiliated Hospital of Harbin Medical University (No.201829). This study was conducted in accordance with the Declaration of Helsinki. We discussed the research protocol and the potential risks with each participant, all participants provided written informed consent. This study has been registered in the Chinese Clinical Trial Registry (Registration number: ChiCTR2000039278).

\section{Data Collection}

A general Health Questionnaire was completed by a trained survey coordinator. Demographic information and past medical history related to migraine were gathered. The major information in this instrument included: 1) age; 2) gender; 3) body height $(\mathrm{cm})$, body weight $(\mathrm{kg})$, and body mass index (BMI), calculated as weight (in $\mathrm{kg}$ )/ height $\mathrm{t}^{2}$ (in $\mathrm{m}^{2}$ ); 4) migraine with or without aura; 5) episodic migraine or chronic migraine; 6) time elapsed since initial migraine diagnosis. To collect the information regarding the headache frequency and average pain intensity, participants were asked to complete the Migraine Disability Assessment questionnaire (MIDAS), with two supplemental questions: "On how many days in the last 3 months did you have a headache (If a headache lasted more than 1 day, count each day)" and "On a scale of $0-10$, on average how painful were these headaches? (Where $0=$ no pain at all, and $10=$ pain as bad as it can be)" (Visual Analog Scale, VAS). ${ }^{20}$

We also assessed the impact of migraine on participants' daily activity, ability to work, quality of life, depression, anxiety, sleep quality, and gastrointestinal symptoms through the following five questionnaires: 1) Headache Impact Test-6 (HIT-6), scores range from 36 to 78, with higher scores indicating greater adverse impact; 2) Migraine-Specific Quality of Life Questionnaire version 
2.1 (MSQ v2.1), with raw dimension scores ranging from 14 to 84 , higher scores indicating worse health status; ${ }^{21} 3$ ) Self-Rating Anxiety Scale (SAS), with an index score ranging from 25 to 100 , a score $\geq 50$ is defined as experiencing anxiety; 4) Self-Rating Depression Scale (SDS) which has a similar scoring structure as SAS, and an index score $\geq 50$ is defined as experiencing depression; ${ }^{22}$ 5) Pittsburgh Sleep Quality Index (PSQI) with questions related to bed partners removed, a PSQI $\geq 8$ is defined as poor sleep quality; ${ }^{23}$ and 6) Gastrointestinal Symptom Rating Scale (GSRS) ${ }^{24}$ Participants' dermatosis was diagnosed by an experienced dermatologist.

\section{Sample Collection and Measurements Blood Sample}

A fasting $5 \mathrm{~mL}$ venous blood sample was drawn from an antecubital vein from each participant, using serum separator tube. The whole undisturbed blood samples were allowed to clot at room temperature for two hours, and were then centrifuged for $20 \mathrm{~min}$ at $1000 \times \mathrm{g}$. The supernatant of the serum was stored at -80 degrees Celsius.

\section{Food Specific IgG Antibodies}

We used enzyme-linked immunosorbent assays (ELISA) to measure the concentration of 14 food specific IgG antibodies (Bioeurope $\mathrm{GmbH}$, Germany). A standard curve was generated for each targeted $\operatorname{IgG}$ antibody by plotting the mean absorbance as the $\mathrm{Y}$ axis against the $\operatorname{IgG}$ concentration as $\mathrm{X}$ axis. The procedure was optimized by using negative control, the concentration of $\operatorname{IgG}$ was calculated based on the standard curve. According to the manufacturer's recommendations, all concentrations $\geq 50 \mathrm{u} / \mathrm{mL}$ were considered a positive reaction to a certain food. Based on the results, participants were assigned to the IgG positive group if at least one food specific IgG antibody was positive, otherwise, they were assigned to the $\mathrm{IgG}$ negative group. Each participant assigned to the IgG positive group was categorized into subgroups, one positive food allergen, two positive food allergens, and three or more positive food allergens. IgG concentrations were summed up as a total positive $\operatorname{IgG}$, if any $\operatorname{IgG}$ concentration $\geq 50 \mathrm{u} / \mathrm{mL}$.

\section{IL-6, IL-10, and TNF- $\alpha$}

The concentrations of IL- 6 , IL-10, and TNF- $\alpha$ were measured using ELISA kits purchased from BIM (San Francisco, CA, Assay range: $1.5 \mathrm{pg} / \mathrm{mL}-48 \mathrm{pg} / \mathrm{mL}$ ), Shanghai Hengyuan Biological Technology Co. Ltd (assay range: $10 \mathrm{ng} / \mathrm{L}-400 \mathrm{ng} / \mathrm{L}$ ), and BIM (San
Francisco, CA, assay range: $2.5 \mathrm{pg} / \mathrm{mL}-80 \mathrm{pg} / \mathrm{mL}$ ), respectively. The procedures were similar as described before. All ELISA assays were performed using Biochrom Anthos 2010 Microplate Reader.

\section{Statistical Analysis}

We checked the distribution, outliers, and missingness for the food specific IgG antibodies, three inflammatory cytokines, and data collected from the questionnaires. Data with highly skewed distributions would be log-transformed to be less skewed data. Descriptive statistics were used to compare between (1) the groups with different numbers of food allergens; and (2) positive and negative IgG groups. The continuous and frequency data were described using mean \pm SD and proportion $(95 \% \mathrm{CI})$, respectively. Chi-squared was used to test the difference of the proportions between groups. For the continuous data, the independent sample $t$-test was conducted if the comparison involved two groups, while ANOVA was used when more than two groups were involved (ie, groups with different number of food allergens).

Consequently, we built a multivariate linear and logistic regression model to further assess the association between each outcome variable of migraine and the related comorbidities with IgG concentration. We then included the three inflammatory cytokines in the regression model to assess the potential mediation effect of the inflammatory cytokines in the association between /IgG levels and migraine/commodities. The odds ratio (OR) and the 95\% CI were reported. The regression analysis was only performed within the group with positive IgG. Data were analyzed using SAS 9.4. ${ }^{25}$

\section{Results}

The sample comprised of 89 eligible participants, the majority was female (60 vs 29 ) with a mean age of 40.3 years $(\mathrm{SD}=9.2)$. Average BMI was $23.5(\mathrm{SD}=3.7)$. The time elapsed since initial diagnosis ranged from 12 to 540 months, the median time was 72 months, with $12.4 \%$ of the patients reporting chronic headache.

\section{Serum Food Specific IgG Antibodies}

IgG antibodies, of varying concentrations, were detected in all participants, 22 of whom were assigned to the $\mathrm{IgG}$ negative group, with no food specific IgG concentrations $\geq 50 \mathrm{u} / \mathrm{mL}$ (24.7\%) and 67 participants had at least one food specific $\operatorname{IgG} \geq$ $50 \mathrm{u} / \mathrm{mL}$ and were thus classified to the $\operatorname{IgG}$ positive group (75.3\%). Participants with one, two, and three or more positive food specific IgG antibodies accounted for 36 (53.7\%), 23 (34.3\%), and $8(11.9 \%)$ positive IgG participants, respectively. 
We found that the top two most prevalent food allergens were eggs and cow's milk. Among the 67 participants in the positive group, $43.3 \%$ had positive egg specific IgG and $26.9 \%$ had positive cow's milk specific IgG. While rice was found to have the lowest frequency of positive cases (Figure 1). For participants in the positive IgG group, total positive IgG concentrations ranged from 54.3 to $577.4 \mathrm{U} / \mathrm{mL}$, with a mean of $212.4 \mathrm{U} /$ $\mathrm{mL}(\mathrm{STD}=153.9 \mathrm{U} / \mathrm{mL})$, and a median of $178.5 \mathrm{U} / \mathrm{mL}$.

\section{Characteristics Between the IgG Positive and Negative Groups, and Groups with Different Numbers of Food Allergens}

Demographic characteristics, migraine characteristics, migraine-related comorbidities, and inflammatory cytokines were compared between the different IgG groups, as seen in Table 1. Demographic characteristics did not significantly differ between the IgG positive and negative groups, nor among the three IgG positive subgroups. Compared to the negative group the time elapsed since diagnosis was significantly longer, additionally, the scores of MIDAS, HIT-6, MSQ, GSRS and SAS were higher for the participants in the IgG positive group. This relationship was also seen with the concentrations of IL- 6 and TNF- $\alpha$, with a higher concentration seen among the IgG positive group. This consistent pattern was observed within the IgG positive group, that is, subgroups with more food allergens generally had worse conditions of migraine (including chronic migraine and the frequency of the migraine attacks), GSRS scores and higher levels of IL- 6 and TNF- $\alpha$.

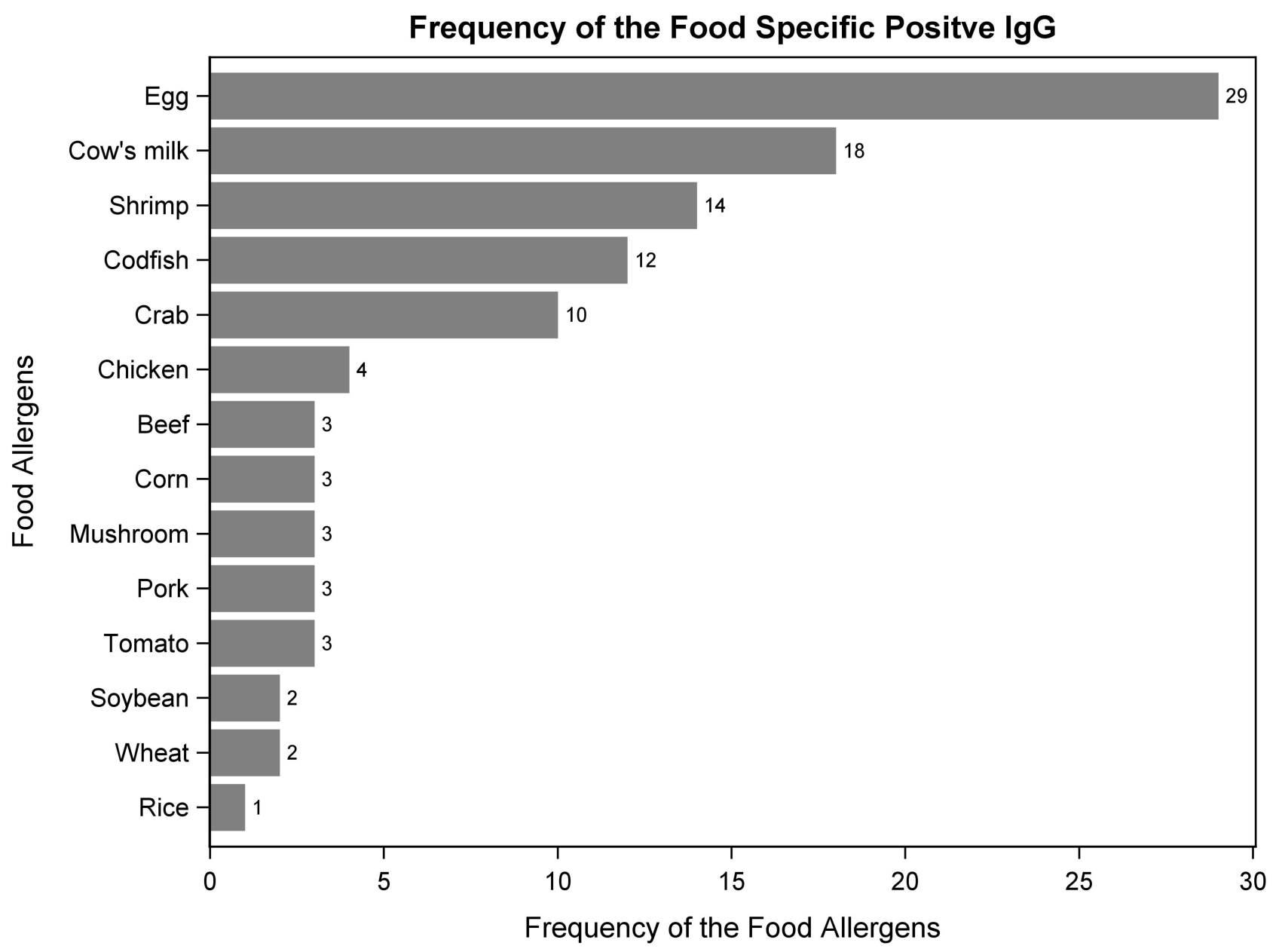

Figure I The frequency of the allergenic foods among 67 participants with positive IgG. 
Table I Comparison of the Participants' Characteristics Between Different Food Specific IgG Groups

\begin{tabular}{|c|c|c|c|c|c|c|c|}
\hline \multirow[t]{2}{*}{ Characteristics $^{\mathbf{a}}$} & \multirow{2}{*}{$\begin{array}{c}\text { IgG Negative } \\
(n=22)\end{array}$} & \multicolumn{5}{|c|}{ IgG Positive } & \multirow[t]{2}{*}{$p$ value $^{c}$} \\
\hline & & $\begin{array}{l}\text { Overall } \\
(n=67)\end{array}$ & $\begin{array}{l}\text { With I } \\
\text { Allergen } \\
(n=36)\end{array}$ & $\begin{array}{l}\text { With } 2 \\
\text { Allergens } \\
(n=23)\end{array}$ & $\begin{array}{c}\text { With } \geq 3 \\
\text { Allergens } \\
(n=8)\end{array}$ & $p$ value $^{b}$ & \\
\hline Age & $42.7(10.4)$ & $39.6(8.8)$ & $41.3(9.6)$ & $38.3(7.6)$ & $35.8(6.8)$ & 0.19 & 0.21 \\
\hline Gender (\%Male) & $45.5 \%$ & $28.4 \%$ & $30.6 \%$ & $34.8 \%$ & 0 & 0.18 & 0.19 \\
\hline BMI $\left(\mathrm{kg} / \mathrm{m}^{2}\right)$ & $22.7(2.8)$ & $23.8(3.9)$ & 24.1 (3.7) & 23.1 (3.9) & $24.6(4.6)$ & 0.53 & 0.17 \\
\hline With aura & $4.6 \%$ & $16.4 \%$ & $8.3 \%$ & $34.8 \%$ & 0 & 0.017 & 0.28 \\
\hline Chronic migraine & $4.6 \%$ & $14.9 \%$ & $8.3 \%$ & $13.0 \%$ & $50 \%$ & 0.023 & 0.28 \\
\hline $\begin{array}{l}\text { Time elapsed since diagnosis } \\
\text { (months) }\end{array}$ & $61.0(44.1)$ & $107.8(94.4)$ & $109.7(113.5)$ & $107.8(70.3)$ & $99.5(63.6)$ & 0.96 & 0.0024 \\
\hline MIDAS & $33.3(15.6)$ & $50.5(35.7)$ & $40.6(23.1)$ & 57.1 (37.2) & 76.3 (59.9) & 0.019 & 0.0023 \\
\hline $\begin{array}{l}\text { Days with migraine in past } 12 \\
\text { weeks }\end{array}$ & I5.5 (I7.2) & $22.0(19.4)$ & I7.I (13.2) & $22.1(16.7)$ & $44.0(33.5)$ & 0.0011 & 0.14 \\
\hline VAS & $6.5(1.5)$ & $7.1(1.3)$ & $6.9(1.5)$ & $7.2(1.2)$ & $7.5(1.1)$ & 0.37 & 0.13 \\
\hline HIT-6 & $57.9(5.5)$ & $61.7(6.1)$ & $59.9(6.5)$ & $63.1(4.5)$ & $65.8(6.0)$ & 0.019 & 0.0095 \\
\hline $\begin{array}{l}\text { Days with migraine in past } 4 \\
\text { weeks }\end{array}$ & $4.9(5.3)$ & $7.5(6.5)$ & $6.3(4.9)$ & $6.9(5.1)$ & | 4.6 (II.5) & 0.0030 & 0.070 \\
\hline MSQ & $25.2(11.3)$ & $32.5(12.8)$ & $28.6(11.6)$ & $35.4(12.8)$ & $41.4(12.5)$ & 0.013 & 0.015 \\
\hline GSRS & $26.9(6.4)$ & $31.6(11.0)$ & $28.3(8.1)$ & 31.7 (8.9) & 45.9 (16.7) & $<0.0001$ & 0.018 \\
\hline SDS & $40.1(10.7)$ & $43.5(11.0)$ & $42.6(9.5)$ & $43.1(10.7)$ & $48.8(17.2)$ & 0.36 & 0.21 \\
\hline Depression (\%) & $18.2 \%$ & $28.4 \%$ & $22.2 \%$ & $30.4 \%$ & $50 \%$ & 0.25 & $0.4 I$ \\
\hline SAS & $38.8(7.7)$ & $43.2(10.5)$ & $42.0(8.1)$ & $43.7(9.3)$ & $47.5(20.2)$ & 0.39 & 0.039 \\
\hline Anxiety (\%) & $13.6 \%$ & $23.9 \%$ & $16.7 \%$ & $34.8 \%$ & $25 \%$ & 0.33 & 0.38 \\
\hline PSQI & $7.4(3.5)$ & $8.0(3.5)$ & $8.1(3.0)$ & $7.7(3.5)$ & $8.5(5.3)$ & 0.84 & 0.50 \\
\hline Poor sleeping (\%) & $45.5 \%$ & $46.3 \%$ & $50 \%$ & $43.5 \%$ & $37.5 \%$ & 0.83 & 1.0 \\
\hline Dermatosis (\%) & $22.7 \%$ & $37.9 \%$ & $27.8 \%$ & $63.6 \%$ & $12.5 \%$ & 0.0081 & 0.30 \\
\hline IL-6 (pg/mL) & $8.6(3.8)$ & $12.6(7.1)$ & $10.0(5.1)$ & I $3.8(5.2)$ & $20.5(11.9)$ & 0.0002 & 0.0013 \\
\hline IL-10 (ng/L) & $173.2(78.3)$ & I36.4 (96.0) & $159.7(95.2)$ & 121.0 & $75.9(51.0)$ & 0.050 & 0.078 \\
\hline TNF- $\alpha$ (pg/mL) & $12.0(5.4)$ & $20.3(12.1)$ & $15.3(6.4)$ & 23.5 (II.7) & $33.5(19.5)$ & $<0.0001$ & $<0.0001$ \\
\hline
\end{tabular}

Notes: ${ }^{2}$ Numeric data were described using mean (STD) and categorical data were described by proportion. ${ }^{b} p$ values were obtained from ANOVA global test and Fisher's exact test for the numeric and categorical data, respectively, for comparing the difference among three subgroups with different numbers of food specific allergens. ${ }^{c} p$ values were from two independent $t$-tests and Fisher's exact test for the continuous and categorical data, respectively, for comparing the difference between the lgG positive group vs lgG negative group.

Abbreviations: BMI, body mass index; MIDAS, Migraine Disability Assessment questionnaire; VAS, Visual Analog Scale; HIT-6, Headache Impact Test-6; MSQ, MigraineSpecific Quality of Life Questionnaire; GSRS, Gastrointestinal Symptom Rating Scale; SDS, Self-Rating Depression Scale; SAS, Self-Rating Anxiety Scale; PSQI, Pittsburgh Sleep Quality Index; IL-6, interleukin-6; IL-10, interleukin-10; TNF- $\alpha$, tumor necrosis factor- $\alpha$.

Characteristics of the lgG Positive Group, and the Association Between Migraine and Comorbidities and the Overall Positive IgG Concentration Before and After Adjusting for the Inflammatory Cytokines

Significant positive associations were observed between the IgG concentration and MIDAS score $(\mathrm{p}<0.0001)$, number of days with a migraine in the previous 12 and 4 weeks $(p<0.0001$ and $p=0.0001)$, and GSRS scores $(p=0.0002)$. With the exception of the GSRS score, after adjusting for the ratio of IL- 6 to IL-10, and TNF- $\alpha$, the strength of the association between the IgG level and each of the outcomes previously mentioned tended to decrease. In other words, the coefficients of the IgG concentration were reduced. Higher IgG was also found to be related to slightly higher odds of having chronic migraine instead of episodic migraine $(\mathrm{OR}=$ $1.08, \mathrm{p}=0.0015)$. This association became non-significant 
after including inflammatory factors in the model, though the OR remained relatively unchanged (Table 2).

\section{Discussion}

This is the first clinical evidence about food allergy mediated by food specific IgG antibodies of migraine patients in northern China, all 89 participants in this study were from Harbin, which is the capital city with the highest latitude in China. Milk and eggs were found to have the highest IgG antibodies positive rate in the study, these foods are included in the daily diet of local residents. These animal products have strong sensitization, along with being exposed in early life and frequent consumption, leads to the ability to accumulate high levels of serum IgG antibodies in the body and produce chronic antigen antibody reactions. ${ }^{26}$

This study was the first to evaluate the effect of food allergy mediated by food specific IgG antibodies on migraine and its comorbidities by analyzing the numbers of IgG positive foods and overall positive IgG levels. The consumption of sensitized allergenic food triggers specific $\operatorname{IgG}$ antibodies that are produced by the immune system; those combined with the allergens form an immune complex, this process could activate multisystem inflammatory reactions, ${ }^{27}$ including migraine, depression, anxiety, sleep disorders, irritable bowel syndrome, eosinophilic GI diseases (EGIDs), allergic proctocolitis (AP), urticaria, and eczema. ${ }^{28,29}$

Some scholars have proposed the potential use of IgG as a biomarker to identify foods linked to an increased inflammatory response, which in turn may be linked to migraine headaches. $^{12}$ The hyperinflammatory state is enhanced, manifested by the increased concentration of proinflammatory cytokines, which promotes the activation of the trigeminal nerve. A growing body of evidence has shown that intracranial meningeal neuroinflammation is

Table 2 The Association Between Migraine and Comorbidities and the Overall Positive IgG Concentration Before and After Adjusting for the Inflammatory Cytokines

\begin{tabular}{|c|c|c|c|c|}
\hline \multirow[t]{2}{*}{ Migraine and Comorbidities } & \multicolumn{4}{|c|}{ Coefficient $(95 \% \mathrm{Cl})^{\mathrm{a}}$} \\
\hline & $\begin{array}{c}\text { Not Adjusting for IL-6, IL-I0, and } \\
\text { TNF- } \alpha\end{array}$ & $p$ value & $\begin{array}{c}\text { Adjusting for IL-6, IL-I0, and } \\
\text { TNF- } \alpha\end{array}$ & $p$ value \\
\hline $\begin{array}{l}\text { Time elapsed since diagnosis } \\
\text { (months) }\end{array}$ & $0.58(-0.94,2.09)$ & 0.45 & $0.36(-2.26,2.99)$ & 0.78 \\
\hline MIDAS & $1.31(0.84,1.78)$ & $<0.000$ I & $0.63(-0.17,1.42)$ & 0.12 \\
\hline $\begin{array}{l}\text { Days with migraine in past } 12 \\
\text { weeks }\end{array}$ & $0.70(0.44,0.96)$ & $<0.0001$ & $0.53(0.14,0.91)$ & 0.0083 \\
\hline VAS & $0.002(-0.019,0.023)$ & 0.85 & $0.01 \mathrm{I}(-0.026,0.048)$ & 0.55 \\
\hline HIT-6 & $0.084(-0.011,0.18)$ & 0.083 & $0.0049(-0.16,0.17)$ & 0.95 \\
\hline Days with migraine in past 4 weeks & $0.19(0.099,0.29)$ & 0.0001 & $0.15(0.0074,0.29)$ & 0.04 \\
\hline MSQ & $0.18(-0.020,0.38)$ & 0.077 & $-0.022(-0.36,0.31)$ & 0.89 \\
\hline GSRS & $0.31(0.16,0.47)$ & 0.0002 & $0.36(0.11,0.62)$ & 0.0053 \\
\hline SDS & $0.17(-0.0006 \mathrm{I}, 0.34)$ & 0.051 & $0.16(-0.13,0.44)$ & 0.27 \\
\hline SAS & $0.14(-0.024,0.31)$ & 0.093 & $0.13(-0.12,0.38)$ & 0.32 \\
\hline \multirow[t]{3}{*}{ PSQI } & $-0.0084(-0.064,0.047)$ & 0.76 & $0.01(-0.084,0.10)$ & 0.83 \\
\hline & \multicolumn{4}{|c|}{ OR $(95 \% \mathrm{Cl})^{\mathrm{a}}$} \\
\hline & $\begin{array}{c}\text { Not Adjusting for IL-6, IL-I0, and } \\
\text { TNF- } \alpha\end{array}$ & $p$ value & $\begin{array}{c}\text { Adjusting for IL-6, IL-I0, and } \\
\text { TNF- } \alpha\end{array}$ & $p$ value \\
\hline With aura & $1.003(0.96,1.05)$ & 0.91 & $1.00(0.93,1.07)$ & 0.97 \\
\hline Chronic migraine & $1.08(1.03,1.13)$ & 0.0015 & $1.07(0.99,1.16)$ & 0.099 \\
\hline Depression & $1.03(0.99,1.06)$ & 0.11 & $0.99(0.93,1.06)$ & 0.75 \\
\hline Anxiety & I.0I $(0.98,1.05)$ & 0.48 & $0.98(0.91,1.06)$ & 0.59 \\
\hline Poor sleeping & $0.98(0.95,1.01)$ & 0.22 & I.0I $(0.95,1.07)$ & 0.84 \\
\hline Dermatosis & I.0I $(0.98,1.05)$ & 0.42 & $0.99(0.94,1.05)$ & 0.75 \\
\hline
\end{tabular}

Note: ${ }^{a}$ Associations were all reported as every $10 \mathrm{u} / \mathrm{mL}$ increase in the overall positive $\lg$ level.

Abbreviations: MIDAS, Migraine Disability Assessment questionnaire; VAS, Visual Analog Scale; HIT-6, Headache Impact Test-6; MSQ, Migraine-Specific Quality of Life Questionnaire; GSRS, Gastrointestinal Symptom Rating Scale; SDS, Self-Rating Depression Scale; SAS, Self-Rating Anxiety Scale; PSQI, Pittsburgh Sleep Quality Index; IL-6, interleukin-6; IL-10, interleukin-10; TNF- $\alpha$, tumor necrosis factor- $\alpha$. 
a crucial factor leading to the increased sensitivity of trigeminal nociceptors in migraine patients. ${ }^{30,31}$ In this study, migraineurs with an increased number of positive food allergens and/or higher overall positive IgG concentration had poorer disability measurements and greater related functional impairments, quality of life, disability questionnaire results, and slightly greater odds of having chronic migraine, likely related to inflammation triggered by food specific IgG antibodies and a variety of inflammatory factors produced in this process.

We also found that the GSRS score of the IgG positive group was higher than that for the $\operatorname{IgG}$ negative group. Moreover, a significant positive association was observed between not only MIDAS scores but also GSRS scores and the positive IgG concentration. After adjusting for the ratio of IL- 6 to IL-10, and TNF- $\alpha$, the coefficients of the positive $\operatorname{IgG}$ concentration were reduced. Migraine can often be accompanied by aura of the gastrointestinal (GI) and autonomic nervous symptoms, such as nausea and vomiting. Previous studies on the relationship between migraine and GI disease found that GI disorders have high comorbidity with migraine. ${ }^{3,32}$ Mechanisms explaining how the gut and the brain may interact in patients with migraine may be explained by the gut-brain axis. The interaction between the gut and the brain seems to be influenced by multiple factors such as inflammatory mediators (IL-1 $\beta$, IL-6, IL-8, and TNF- $\alpha$ ), gut microbiota profile, and nutritional substances. ${ }^{33}$ A clinical trial in patients with migraine and irritable bowel syndrome also confirms this view. Food specific allergens were identified by measuring the serum IgG antibodies, then an elimination diet was implemented to avoid the identified allergenic foods, removal of these foods resulted in the alleviation of migraine and IBS disease symptoms. ${ }^{13}$

In addition to migraine conditions, anxiety index scores (SAS) in the positive IgG group were significantly higher than that in negative IgG group, along with a higher prevalence of anxiety disorders. Rammohan et al proposed that the association between migraine, psychiatric comorbidities, and sleep disorders might be explained by the migraine neuro-limbic theory, which suggests that the central sensitization process might be responsible for the threshold changes in pain sensibility. Long-term, repeated, severe migraine attacks, anxiety, depression, sleep disorders, and other mental and psychological symptoms are mutually causal and reinforcing, promoting a vicious cycle. $^{2}$ Nevertheless, our study found an unanticipated nonsignificant difference in depression and sleep disorder between the IgG positive and negative groups. Mercante et al reported nearly half of their subjects presented with a triple diagnosis of anxiety, primary headaches, and depression. The results found supported the progressive disorder model in which anxiety disorders, often at a young age, proceeds to migraine and is followed by depressive episodes in adulthood. ${ }^{34}$ Therefore, we speculate that more promising results may be obtained with longer observation time and more migraine cases.

Additionally, we observed that participants in the IgG positive group were more likely to experience dermatosis than the $\mathrm{IgG}$ negative group, though these differences were not statistically significant. The "aseptic inflammation" state of food allergy mediated by IgG may lead to the release of neuropeptides and inflammatory factors, leading to vasodilation, plasma extravasation secondary to capillary leakage, edema, and mast cell degranulation, ${ }^{30}$ resulting in allergyrelated skin diseases such as urticaria and eczema.

In this study, levels of IL- 6 and TNF- $\alpha$ in the IgG positive group were higher than those in the IgG negative group, the association between IgG and MIDAS lost the significance after including the ratio of IL- 6 to IL-10 and TNF- $\alpha$ in the model, which indicated that these three inflammatory cytokines might partially mediate the causal pathway between food specific IgG and migraine frequency/intensity. Cytokines play an important role in the inflammation process and mediation of pain sensation of migraine patients. These have been reported to be involved in the modulation of pain threshold, by promoting or suppressing the sensitization of trigeminal nerve fibers. ${ }^{18,35}$ A number of studies have found a pro-inflammatory state in chronic migraine and episodic migraine patients, compared with the healthy controls, which has been demonstrated by an increased concentration of proinflammatory cytokines including TNF- $\alpha$ and IL- $6 .{ }^{16,17}$ Togha et al also noted that headache is more likely to be chronic with increased levels of inflammatory factors. ${ }^{17} \mathrm{We}$ hypothesized that increased levels of pro-inflammatory cytokines such as TNF- $\alpha$ and IL- 6 are probable indicators of a low grade chronic inflammatory state caused by $\mathrm{IgG}$ mediated food allergy.

However, there was no significant difference in the level of IL-10 between the two groups, despite the IgG positive group averaging a lower IL-10. In fact, the level of IL-10 in migraine patients is inconsistent with previously published studies. Perini et al found that the plasma concentration of IL10 in migraine patients was similar to that in healthy participants, ${ }^{36}$ but other research reported lower IL-10, compared to healthy participants. ${ }^{37,38}$ Oliveira et al suggested that 
fluctuating IL-10 levels may be associated with head pain and higher psychiatric comorbidities /symptoms. IL-10 is an effective inhibitor of pro-inflammatory cytokines, which can promote antinociception and prevent depressive-like/anxiety behaviors. In addition, IL-10 is a cytokine produced by helper T2 cells (Th2), and is negatively regulated by TNF- $\alpha$ and IL-12. ${ }^{39}$

Our study had strengths but also limitations. First, participants were enrolled during late fall and winter time. Corresponding low temperatures during this time of year result in differing fresh food sources, food processing, and cooking methods than during the spring and summer months. Moreover, our study found a higher prevalence of depression among participants than other studies, ${ }^{2,40}$ presumably resulting from the potential adverse impact of the fall and winter seasons on depression severity. ${ }^{41}$ Second, the sample size for this exploratory study may not be sufficient, potentially compromising the power to detect a statistically significant correlation. The insufficient power was reflected by the lack of significant difference in the prevalence of sleep disorders and dermatosis between the IgG positive and negative groups, while the effect sizes for these two measurements were scientifically meaningful. Third, this study was a crosssectional study and thus could not establish the direct casualty between diet and migraine. We expect to conduct an interventional study in the future to explore the effect of eliminating $\mathrm{IgG}$ positive foods on migraine and the related comorbidities.

\section{Conclusion}

This study shows that positive food specific IgG antibodies seem to be related to migraine and its comorbidities, and inflammatory cytokines might partially impact this process. Further studies are warranted to reveal the immunological pathogenesis of migraine and the effect of dietary modification on migraine.

\section{Abbreviations}

IgG, immunoglobulin G; BMI, body mass index; MIDAS, Migraine Disability Assessment questionnaire; VAS, Visual Analog Scale; HIT-6, Headache Impact Test-6; MSQ, Migraine-Specific Quality of Life Questionnaire; GSRS, Gastrointestinal Symptom Rating Scale; SDS, SelfRating Depression Scale; SAS, Self-Rating Anxiety Scale; PSQI, Pittsburgh Sleep Quality Index; IL-6, interleukin-6; IL-10, interleukin-10; TNF- $\alpha$, tumor necrosis factor- $\alpha$; ELISA, enzyme linked immunosorbent assay.

\section{Data Sharing Statement}

Data can be requested from the corresponding author by E-mail after publication.

\section{Acknowledgments}

We would like to thank all the patients who voluntarily participated in this study, and the laboratory technicians for helping with specimen collection and carrying out the testing.

\section{Author Contributions}

All authors made a significant contribution to the work reported, whether that is in the conception, study design, execution, acquisition of data, analysis and interpretation, or in all these areas; took part in drafting, revising or critically reviewing the article; gave final approval of the version to be published; have agreed on the journal to which the article has been submitted; and agree to be accountable for all aspects of the work.

\section{Funding}

Scientific Research Project of Heilongjiang Provincial Health Commission (No. 2017-042), Research and Innovation Fund of the First Affiliated Hospital of Harbin Medical University (No. 2018Y015), and National Key R\&D Program of China (No. 2017YFC0907405 to YZ).

\section{Disclosure}

The authors report no conflicts of interest in this work.

\section{References}

1. Headache Classification Committee of the International Headache Society (IHS). The international classification of headache disorders, 3rd edition. Cephalalgia. 2018;38(1):1-211. doi:10.1177/ 0333102417738202

2. Rammohan K, Mundayadan SM, Das S, Shaji CV. Migraine and mood disorders: prevalence, clinical correlations and disability. $J$ Neurosci Rural Pract. 2019;10(1):28-33. doi:10.4103/jnrp.jnrp_146_18

3. Talafi Noghani M, Namdar H. Migraine associated with gastrointestinal disorders: a pathophysiological explanation. Med Hypotheses. 2019;125:90-93. doi:10.1016/j.mehy.2019.02.041

4. Han SM, Kim KM, Cho S-J, et al. Prevalence and characteristics of cutaneous allodynia in probable migraine. Sci Rep. 2021;11(1):2467. doi:10.1038/s41598-021-82080-z

5. Merikangas KR. Contributions of epidemiology to our understanding of migraine. Headache. 2013;53(2):230-246. doi:10.1111/head.12038

6. Zaeem Z, Zhou L, Dilli E. Headaches: a review of the role of dietary factors. Curr Neurol Neurosci Rep. 2016;16(11):101. doi:10.1007/ s11910-016-0702-1

7. Gazerani P. Migraine and diet. Nutrients. 2020;12(6):1658. doi: $10.3390 /$ nu12061658 
8. Hammond C, Lieberman JA. Unproven diagnostic tests for food allergy. Immunol Allergy Clin North Am. 2018;38(1):153-163. doi:10.1016/j.iac.2017.09.011

9. Drisko J, Bischoff B, Hall M, McCallum R. Treating irritable bowel syndrome with a food elimination diet followed by food challenge and probiotics. J Am Coll Nutr. 2006;25(6):514-522. doi:10.1080/ 07315724.2006.10719567

10. Tai S, Yap JF, Goh C. Dietary trigger factors of migraine and tension-type headache in a South East Asian country. J Pain Res. 2018;11:1255-1261. doi:10.2147/JPR.S158151

11. Razeghi Jahromi S, Ghorbani Z, Martelletti P, Lampl C, Togha M. Association of diet and headache. J Headache Pain. 2019;20(1):106. doi:10.1186/s10194-019-1057-1

12. Geiselman JF. The clinical use of $\mathrm{IgG}$ food sensitivity testing with migraine headache patients: a literature review. Curr Pain Headache Rep. 2019;23(11):79. doi:10.1007/s11916-019-0819-4

13. Aydinlar EI, Dikmen PY, Tiftikci A, et al. IgG-based elimination diet in migraine plus irritable bowel syndrome. Headache. 2013;53 (3):514-525. doi:10.1111/j.1526-4610.2012.02296.x

14. Murphy SF, Schaeffer AJ, Thumbikat P. Immune mediators of chronic pelvic pain syndrome. Nat Rev Urol. 2014;11(5):259-269. doi:10.1038/nrurol.2014.63

15. Cavaillon JM. Pro- versus anti-inflammatory cytokines: myth or reality. Cell Mol Biol. 2001;47(4):695-702.

16. Han D. Association of Serum levels of calcitonin gene-related peptide and cytokines during migraine attacks. Ann Indian Acad Neurol. 2019;22(3):277-281. doi:10.4103/aian.AIAN_371_18

17. Togha M, Razeghi Jahromi S, Ghorbani Z, Ghaemi A, Rafiee P. Evaluation of inflammatory state in migraineurs: a Case-control Study. Iran J Allergy Asthma Immunol. 2020;19(S1):83-90.

18. Kwilasz AJ, Grace PM, Serbedzija P, Maier SF, Watkins LR. The therapeutic potential of interleukin-10 in neuroimmune diseases. Neuropharmacology. 2015;96(Pt A):55-69. doi:10.1016/j. neuropharm.2014.10.020

19. Martami F, Razeghi Jahromi S, Togha M, Ghorbani Z, Seifishahpar M, Saidpour A. The serum level of inflammatory markers in chronic and episodic migraine: a case-control study. Neurol Sci. 2018;39(10):1741-1749. doi:10.1007/s10072-018-3493-0

20. Hung PH, Fuh JL, Wang SJ. [The application of migraine disability assessment questionnaire (MIDAS)]. Acta Neurol Taiwan. 2006;15 (1):43-48. Italian.

21. Rendas-Baum R, Bloudek LM, Maglinte GA, Varon SF. The psychometric properties of the Migraine-Specific Quality of life questionnaire version 2.1 (MSQ) in chronic migraine patients. Qual Life Res. 2013;22(5):1123-1133. doi:10.1007/s11136-012-0230-7

22. Dunstan DA, Scott N. Assigning clinical significance and symptom severity using the zung scales: levels of misclassification arising from confusion between index and raw scores. Depress Res Treat. 2018;2018:9250972.

23. Buysse DJ, Reynolds CF 3rd, Monk TH, Berman SR, Kupfer DJ. The pittsburgh sleep quality index: a new instrument for psychiatric practice and research. Psychiatry Res. 1989;28(2):193-213. doi:10.1016/0165-1781(89)90047-4

24. Svedlund J, Sjödin I, Dotevall G. GSRS-a clinical rating scale for gastrointestinal symptoms in patients with irritable bowel syndrome and peptic ulcer disease. Dig Dis Sci. 1988;33(2):129-134. doi:10.1007/BF01535722
25. SAS 9.4. Computer Program. Cary, NC: SAS Institute Inc.2016.

26. Zeng Q, Dong SY, Wu LX, et al. Variable food-specific IgG antibody levels in healthy and symptomatic Chinese adults. PLoS One. 2013;8 (1):e53612. doi:10.1371/journal.pone.0053612

27. Shakoor Z, AlFaifi A, AlAmro B, AlTawil LN, AlOhaly RY. Prevalence of IgG-mediated food intolerance among patients with allergic symptoms. Ann Saudi Med. 2016;36(6):386-390. doi:10.5144/0256-4947.2016.386

28. Boyce JA, Assa'ad A, Burks AW, et al. Guidelines for the diagnosis and management of food allergy in the United States: report of the NIAID-sponsored expert panel. J Allergy Clin Immunol. 2010;126(6 Suppl):S1-S58. doi:10.1016/j.jaci.2010.10.008

29. Gaby A. Nutritional Medicine. 2nd ed. Concord, NH: Fritz Perlberg Publishing; 2013.

30. Ramachandran R. Neurogenic inflammation and its role in migraine. Semin Immunopathol. 2018;40(3):301-314. doi:10.1007/s00281-0180676-y

31. Malhotra R. Understanding migraine: potential role of neurogenic inflammation. Ann Indian Acad Neurol. 2016;19(2):175-182. doi:10.4103/0972-2327.182302

32. Lee SH, Lee JJ, Kwon Y, Kim JH, Sohn JH. Clinical Implications of associations between headache and gastrointestinal disorders: a study using the hallym smart clinical data warehouse. Front Neurol. 2017;8:526. doi:10.3389/fneur.2017.00526

33. Arzani M, Jahromi SR, Ghorbani Z, et al. Gut-brain axis and migraine headache: a comprehensive review. J Headache Pain. 2020;21(1):15. doi:10.1186/s10194-020-1078-9

34. Mercante JP, Peres MF, Bernik MA. Primary headaches in patients with generalized anxiety disorder. J Headache Pain. 2011;12 (3):331-338. doi:10.1007/s10194-010-0290-4

35. Milligan ED, Penzkover KR, Soderquist RG, Mahoney MJ. Spinal interleukin-10 therapy to treat peripheral neuropathic pain. Neuromodulation. 2012;15(6):520-526; discussion 526. doi:10.1111/ j.1525-1403.2012.00462.x

36. Perini F, D’Andrea G, Galloni E, et al. Plasma cytokine levels in migraineurs and controls. Headache. 2005;45(7):926-931. doi:10.1111/j.1526-4610.2005.05135.x

37. Boćkowski L, Smigielska-Kuzia J, Sobaniec W, ZelazowskaRutkowska B, Kułak W, Sendrowski K. Anti-inflammatory plasma cytokines in children and adolescents with migraine headaches. Pharmacol Rep. 2010;62(2):287-291. doi:10.1016/S1734-1140(10) 70268-1

38. Uzar E, Evliyaoglu O, Yucel Y, et al. Serum cytokine and pro-brain natriuretic peptide (BNP) levels in patients with migraine. Eur Rev Med Pharmacol Sci. 2011;15(10):1111-1116.

39. Oliveira AB, Bachi ALL, Ribeiro RT, Mello MT, Tufik S, Peres MFP. Unbalanced plasma TNF- $\alpha$ and IL-12/IL-10 profile in women with migraine is associated with psychological and physiological outcomes. J Neuroimmunol. 2017;313:138-144. doi:10.1016/j. jneuroim.2017.09.008

40. Lampl C, Thomas H, Tassorelli C, et al. Headache, depression and anxiety: associations in the Eurolight project. $J$ Headache Pain. 2016;17(1):59. doi:10.1186/s10194-016-0649-2

41. Zauderer C, Ganzer C. Seasonal affective disorder: an overview: Cheryl Zauderer and $\mathrm{C}$ Anne Ganzer outline the diagnosis, causes and treatment options for people who experience the winter blues. Ment Health Pract. 2015;18(9):21-24. doi:10.7748/mhp.18.9.21.e973 
Journal of Pain Research

\section{Publish your work in this journal}

The Journal of Pain Research is an international, peer reviewed, open access, online journal that welcomes laboratory and clinical findings in the fields of pain research and the prevention and management of pain. Original research, reviews, symposium reports, hypothesis formation and commentaries are all considered for publication. The manuscript

Submit your manuscript here: https://www.dovepress.com/journal-of-pain-research-journ management system is completely online and includes a very quick and fair peer-review system, which is all easy to use. Visit http:// www.dovepress.com/testimonials.php to read real quotes from published authors. 